\title{
Un train peut en cacher un autre
}

L'entre-deux-guerres de Romain Rolland en Suisse (1922-1938)

Jean-Pierre Meylan

\section{CpenEdition}

\section{Journals}

Édition électronique

URL : http://journals.openedition.org/edl/338

DOI : 10.4000/edl.338

ISSN : 2296-5084

Éditeur

Université de Lausanne

\section{Édition imprimée}

Date de publication : 15 septembre 2012

Pagination : $29-48$

ISBN : 978-2-940331-28-4

ISSN : 0014-2026

\section{Référence électronique}

Jean-Pierre Meylan, « Un train peut en cacher un autre », Études de lettres [En ligne], 3 | 2012, mis en ligne le 15 septembre 2015, consulté le 22 décembre 2020. URL : http://journals.openedition.org/edl/ 338 ; DOI : https://doi.org/10.4000/edl.338 


\section{UN TRAIN PEUT EN CACHER UN AUTRE. L'ENTRE-DEUX-GUERRES DE ROMAIN ROLLAND EN SUISSE (1922-1938) ${ }^{1}$}

Romain Rolland vécut pendant presque toute la période de l'entre-deux-guerres en Suisse où il pensait trouver, après la catastrophe de la guerre, le loisir nécessaire à la création littéraire et intellectuelle. Villeneuve, proche de Genève, où s'installa la Société des Nations, lui offrait à la fois du calme mais aussi un observatoire neutre dans un réseau dense de relations internationales. De là, il lança son appel Aux esprits libres (1919), embrassa l'idée d'un renouveau intellectuel, politique et social dans une Europe qui sortait d'une banqueroute morale (avec la fondation de la revue Europe) et s'ouvrit au monde asiatique, notamment indien.

Après un calme relatif pour la France, fondé sur la fausse confiance qu'inspirait la victoire, Romain Rolland se rendit rapidement compte que les séquelles de la guerre allaient miner cette paix précaire. Nul ne se doutait encore que la facture allait être à ce point élevée pour tous: cette période allait être dominée par les révolutions (à défaut d'une mondiale, celle de l'URSS), l'éveil de nouveaux nationalismes, la lutte anticoloniale et anti-impérialiste, la dévaluation économique généralisée, la rupture culturelle et des clivages idéologiques sans précédent. Rolland vécut tout cela en Suisse, cette fois non en observateur "au-dessus de la mêlée", mais en acteur de plus en plus "engagé»: et bientôt comme "compagnon de route» du communisme. Car, même s'il créa quelques œuvres

I. Les documents utilisés proviennent des fonds Romain Rolland suivants: Berne, Archives littéraires suisses (également fonds H. Häberlin); Bâle, Universitätsbibliothek; Berne, Archives fédérales; Lausanne, Archives cantonales vaudoises; Villeneuve (VD), Archives communales. 
littéraires importantes à cette époque, il n'échappa pas à l'engrenage de la lutte politique. Un combat parmi d'autres qui domina, fut celui de la «lutte antifasciste» (terme alors non encore instrumentalisé). Le panneau standard de signalisation de la SNCF aux passages à niveau "un train peut en cacher un autre" s'applique bien à Rolland. A force de combattre le danger des fascismes, il n'a pas vu un autre danger, celui du stalinisme, dans les années 30 .

Par malchance la Suisse ne resta pas un havre de paix et de relative sérénité comme entre 1914 et 1918: Rolland y fut témoin de combats idéologiques dramatiques: le pays fut écartelé par la révolution sociale, le combat de la gauche contre la droite, un durcissement idéologique sans précédent et des troubles violents. Rolland ne se mêla jamais de questions internes à la Suisse - observant ainsi le devoir de retenue d'un résidant étranger et vedette publique. Mais l'opinion publique suisse faisait écho des débats en France, si bien que les polémiques débordèrent sur la Suisse. Rolland y devint ici un phare de paix (pour la gauche), là un élément subversif (pour la droite), car depuis son Prix Nobel en 1916, il était devenu une figure publique mondialement commentée. Rolland devint ainsi pour la Suisse romande officielle un hôte dérangeant, dont on aurait bien voulu se défaire s'il n'avait pas été une vedette. L'image de Rolland en France passa de la gauche modérée au stalinisme, tandis que son image en Allemagne et en Suisse alémanique resta longtemps celui d'un pacifiste au-dessus des camps politiques, une figure d'intégration.

Notre propos ici se concentre sur le volet politique de cet entre-deuxguerres de Rolland, raison pour laquelle il ne sera fait que marginalement allusion à d'autres rencontres suisses littéraires importantes, par exemple celle de Carl Spitteler (1845-1924) et d'Edmond Privat (18891962), toutes deux déjà bien documentées ${ }^{2}$. Les deux eurent d'ailleurs des implications politiques non négligeables.

Rolland rencontra Spitteler en 1915, à la suite d'un discours célèbre que celui-ci avait prononcé en faveur de la neutralité de la Suisse et de la réconciliation entre Romands et Alémaniques ${ }^{3}$. Bon connaisseur de la littérature alémanique, il tint son œuvre néo-classique en grande estime. Ce fut d'ailleurs grâce à l'appui entre autres de Rolland que Spitteler

2. Voir J.-P. Meylan, «Romain Rolland au-dessus, mais aussi dans la mêlée».

3. Pour les retombées politiques de la réception de Spitteler, voir F. Vallotton, Ainsi parlait Carl Spitteler et J. Schütt, Germanistik und Politik. 
obtint le Prix Nobel de littérature en 1920. Après la mort de celui-ci, en 1924, cette figure helvétique fut accaparée par la droite conservatrice et autoritaire, bien au déplaisir de Rolland.

Edmond Privat, une génération plus jeune, est issu d'une famille des montagnes neuchâteloises et se caractérise par son attachement à un christianisme socialisant, et également par sa culture imprégnée d'anarcho-syndicalisme et d'individualisme contestataire. Il se fit assez tôt une réputation internationale dans la promotion de l'espéranto et fut un journaliste de renom, un voyageur aux grands horizons comme Blaise Cendrars ou plus tard Nicolas Bouvier. Ce fut lui qui assista Rolland comme une sorte "d'attaché de presse", lorsque le mahatma M. K. Gandhi se rendit en Suisse à Villeneuve. La photo de cette rencontre très médiatisée entre Rolland et Gandhi à la villa Olga, de décembre 1931, fit le tour du monde. Cette péripétie fut bientôt obscurcie par des préoccupations bien plus dramatiques dans le cadre de l'engagement de Rolland en faveur d'un «front anti-fasciste». Ce combat oblitéra dans les années 30 tout un pan du travail de Rolland, considérable d'ailleurs, en faveur du rapprochement entre l'Europe et l'Asie, et plus particulièrement de l'Inde 4 .

La relation entre Rolland et le théâtre, notamment romand, mériterait une étude approfondie. Mentionnons simplement ici l'influence de Rolland sur le théâtre romand, antérieur à 1914, avec René Morax et son Théâtre de Mézières.

\section{Romain Rolland dans une Suisse en convulsions idéologiques}

Rolland revint donc dans une Suisse politiquement bouleversée à la suite de la grève générale de 1918. Cette grève reste un événement mémoriel majeur de l'histoire suisse. Elle fut interprétée comme une révolution bolchevique manquée qui divisa longtemps la Suisse, cette fois non seulement le long du "fossé» entre Romands et Alémaniques, mais entre idéologies politiques. Comme ailleurs, le camp ouvrier se divisa en sociaux-démocrates et en communistes, ces derniers restant longtemps

4. Voir J.-P. Meylan: La «Weltbibliothek» et la Maison des Amis, un projet de Romain Rolland et «Der Plan einer Weltbibliothek von Romain Rolland und seinem Schweizer Verleger und Mäzen Emil Roniger, 1922-1926». 
faibles, sectaires et isolés. Les partis bourgeois furent dépassés à droite par des ligues civiques qui formèrent le Front National (Frontistes) dans les années 30. La droite catholique s'établit à nouveau contre le camp radical et libéral qui avait dominé jusqu'en 1914. Les milieux agraires (protestants et catholiques) affrontaient la Suisse industrielle et touristique. Le canton de Vaud se signala par une réaction agraire virulente dans sa majorité ${ }^{5}$, ce qui ne facilita pas le séjour de Rolland à Villeneuve, au bord du lac, où l'on était plus cosmopolite et où l'on dépendait de la libre circulation nécessaire au tourisme. Le monde politique vaudois de l'entre-deux-guerres contrastait fortement avec la Genève politiquement déchirée, où les échauffourées de 1932 se soldèrent par treize morts.

Un évènement dramatique émut le pays en 1923: un Suisse ex-émigré de Russie, spolié par la révolution, assassina à Lausanne le diplomate soviétique Vorovsky. Il fut acquitté par une cour cantonale après un procès scandaleusement biaisé aux dépens du jeune régime soviétique. Ultérieurement, au cours de la phase staliniste dure, le GPU soviétique n'hésita pas à mandater des assassinats en Suisse, dont de celui d'Ignaz Reiss, en 1937, à Lausanne aussi. Et puis, ce fut dans le canton de Vaud que le jeune mouvement fasciste italien, au gouvernement dès 1922, eut le plus d'adeptes - au point d'amener l'Université de Lausanne à décerner un doctorat honoris causa à Mussolini, en 1937. Au gouvernement fédéral, Giuseppe Motta, "ministre des affaires étrangères" misait sur une bonne entente avec le régime de Mussolini, auquel on attribuait un "visage humain» par rapport au nazisme ${ }^{6}$.

Malgré l'adhésion à la Société des Nations et la présence de celle-ci à Genève, le pays fut gagné par une poussée xénophobe qui eut des conséquences pour la législation fédérale. Paradoxalement, c'est dans les années 20, lorsque le taux de population étrangère baissa de $20 \%$ à $10 \%$ que la hantise de l'étranger augmenta. Une fièvre sécuritaire s'ensuivit et intoxiqua l'atmosphère intellectuelle. Rolland en fit personnellement les frais. Par comparaison, la France politique de cette époque semblait un pays pacifique qui s'était concilié avec l'Allemagne de Weimar (pacte de

5. Avec la construction, en littérature, du mythe "latin», lacustre et alpin de la Suisse romande, opposé à "l'Esprit de Genève», une période d'affirmation de soi fut entamée, au prix d'une régionalisation défensive de la littérature romande qui dura bien au-delà de la Seconde Guerre mondiale.

6. Voir à ce sujet les études d'A. Mattioli, notamment son Gonzague de Reynold. 
Locarno en 1925; la loi sur la naturalisation des étrangers de 1927) et le Front populaire dès 1936 laissait paraître ce pays comme un havre de paix protégeant les victimes des fascismes.

Rolland profita néanmoins de la relative ouverture de l'«Esprit de Genève " ${ }^{7}$ entre 1920 et 1926 pour poursuivre sa recherche de la nonviolence et du commerce intellectuel avec l'Inde. Ce fut le Bâlois Emil Roniger, un de ses éditeurs en langue allemande, le Bengalais Kalidas $\mathrm{Nag}$ et notamment sa sœur Madeleine, très experte en littérature bengali, qui facilitèrent ces rencontres - surtout celle avec Tagore - et les traductions des essais de Rolland sur Gandhi ${ }^{8}$.

On peut donc délimiter deux "périodes suisses" de Rolland: celle des années 20 à 30 - dans la continuité rollandienne du libéralisme d'esprit et de la pensée panthéiste ouverte - et celle des années 30 à 39 - contraire au Rolland de 1914-1918 - obscurcie, voire obnubilée par le combat idéologique, où il fut happé par des organisations qui le dépassèrent et où il devint un "pion sur un échiquier", un de ces "compagnons de route» bientôt désillusionnés, comme les a décrit François Furet dans son Passé d'une illusion.

\section{Romain Rolland aux prises avec la bureaucratie suisse}

Accorder le droit de domicile permanent aux étrangers est affaire des communes ou municipalités en Suisse. Ce fut le cas avant 1918 et l'est encore aujourd'hui, mais depuis les années 20 , ce droit est coiffé d'une législation fédérale qui filtre ces séjours grâce à la nouvelle police fédérale des étrangers de plus en plus restrictive. Comme Français parmi des dizaines de milliers d'autres en Suisse, Rolland n'avait rien à craindre, mais comme philo-soviétique il souffrit de vexations discrètes. Comme tout étranger voulant s'établir en Suisse, il fut l'objet d'un conflit d'intérêts: le canton et la police fédérale des étrangers étaient sourcilleux à l'égard de résidents à haut profil politique qui souvent polarisaient le

7. La Revue de Genève (1920-1930), fondée par Robert de Traz, professa aussi une ouverture vers le monde, mais s'opposa complètement à l'internationalisme «de gauche", celui de Rolland, dont elle ne fit jamais mention.

8. Pour les initiatives de Rolland en vue d'une ouverture vers la culture indienne, voir J.-P. Meylan, La "Weltbibliothek" et la Maison des Amis, un projet de Romain Rolland. 
public ou attiraient dans leur sillon des «indésirables». On surveillait les fascistes italiens les plus actifs, les anarchistes ou libertaires, et les communistes - plus tard les sympathisants nazis. Par exemple, on accorda en 1932 à Henri Barbusse un droit de passage à Lucerne strictement limité à "douze heures sur le territoire suisse», comme s'il s'agissait d'un pestiféré.

Mais la finance, l'industrie et le tourisme dominants, dépendants d'échanges ouverts avec l'étranger, voulaient des frontières ouvertes. C'est le cas pour toute la région de la Riviera lémanique, dont Villeneuve, qui attirait un tourisme général, éducatif (avec ses internats de jeunes filles étrangères bien chaperonnées) et médical (dont Rolland fit beaucoup usage). Et puis, la concurrence fiscale entre cantons et entre municipalités étant féroce, Villeneuve avait tout intérêt à ne pas s'aliéner un contribuable bienvenu. Aujourd'hui encore le canton de Vaud compte un record de résidents étrangers fortunés, forfaitairement taxés, quasiment "à la tête du client». Un procès-verbal du conseil municipal de Villeneuve mentionne qu'en 1936, Rolland avait alors des revenus qui baissaient, mais qu'on ne demanderait pas de justificatifs pour ne pas «incommoder un étranger bienvenu»?

En mai 1926, Rolland reçut un courrier officiel du canton qui le froissa: on lui demanda de remplir un formulaire pour solliciter le droit de domicile permanent, formulaire exigeant à l'appui un certificat de bonnes mours, un extrait de casier judiciaire et des personnes de garantie (cela pour lui-même, pour son père et sa sœur). A lui qui était une vedette internationale et qui séjournait depuis des années en Suisse! Indigné, il s'adressa au chef du Département fédéral de justice et police, Heinz Häberlin ${ }^{10}$, qui, en cette année fut également président de la

9. Détail communiqué par $\mathrm{M}^{\mathrm{me}}$ Michelle Grote, archiviste de la municipalité de Villeneuve.

Io. Häberlin fut un grand juriste thurgovien et radical, auteur du Code Pénal Suisse (avalisé en 1939, cantonal auparavant). Il fut également juge cantonal (TG) et membre du Conseil national (député). Il échoua par deux fois à faire passer une loi sécuritaire, dite «Lex Häberlin» en 1922 et 1934 (chaque fois refusée par référendum populaire). Il fut, de 1920 à 1934, conseiller fédéral (ministre de la Police et de la Justice) et, en 1926 et en 1931, président de la Confédération. Rien ne le prédestinait à devenir un correspondant de Rolland, car il représentait le radicalisme pur et dur. Dès 1934, après le départ de Häberlin, Rolland n'eut plus personne au haut niveau politique pour le protéger: on sent que l'administration fédérale, notamment le Ministère fédéral (Office du Procureur fédéral) ne ménagea plus Rolland. La correspondance Rolland - Häberlin 
Confédération. Häberlin fut - ironie du sort! - précisément le ministre qui mit en place un système de surveillance des étrangers et voulut faire passer au parlement des lois sécuritaires. On pourrait s'imaginer un nonrecevoir : ce ne fut point le cas. Häberlin était un radical thurgovien, un juriste internationalement respecté et ancien juge cantonal, rigoriste - mais un homme de lettres aussi, quoique aux antipodes de la pensée de Rolland. Häberlin expliqua qu'il ne s'agissait aucunement d'un traitement discriminatoire, que tout le monde y était astreint et que luimême avait passé par cette même procédure pour devenir juge. Häberlin amadoua Rolland. Il s'en suivit une correspondance bientôt amicale qui dura jusqu'à la guerre mondiale. Rolland avait gagné en lui un protecteur qui ne lui accordait aucun passe-droit, mais le guidait à travers les vicissitudes de la bureaucratie helvétique. Häberlin dut même s'employer à convaincre, dans un entretien particulier spécialement arrangé à cet effet, le chef du Département de justice et police du canton de Vaud, le conseiller d'Etat Dufour, de lui accorder le droit de domicile - ce qui fut fait du bout des doigts, chaque fois pour des périodes d'une année, renouvelables. Rien ne se fait sans accord d'un canton en Suisse.

Le chef de la sûreté vaudoise (police cantonale) était alors un certain Juillard, qui s'était déjà signalé par une politique sécuritaire énergique lors du procès contre l'assassin de Vorovsky, en 1923. Il fit ultérieurement une carrière militaire qui le conduisit, comme colonel, à la tête des services de contre-espionnage de l'armée pendant la Seconde Guerre mondiale. Ce fut lui qui ne cessa de harceler Rolland derrière les coulisses tout au long des années 30 .

\section{Rolland à nouveau sous surveillance}

Rolland faisait l'objet d'une surveillance constante par la police genevoise et le service de renseignements de l'armée pendant la Première Guerre mondiale: copie des télégrammes, courrier, observation des visites

fut publiée dans P. Abraham et al., Romain Rolland. Le fonds Häberlin aux Archives littéraires suisses et aux Archives cantonales de Thurgovie, à Frauenfeld, contiennent des lettres encore inédites, entre autres avec Marie Romain-Rolland, qui montrent que Häberlin et un comité d'amis suisses avaient préparé le terrain pour un éventuel retour de Rolland à Villeneuve pendant la Seconde Guerre mondiale. 
d'étrangers, etc. En France, à plus forte raison, aussi. La justice militaire française frappa lourdement l'entourage des pacifistes français de Rolland, à défaut de pouvoir s'en prendre à Rolland lui-même. La Suisse romande étant très francophile, on ne s'étonnera pas que le secret administratif à l'égard de la France n'y était pas respecté (pas plus qu'entre la Suisse alémanique et l'Allemagne) : le $2^{\mathrm{e}}$ bureau français à l'ambassade de Berne n'eut aucune peine pour s'alimenter et put constituer des dossiers non seulement sur Rolland, mais aussi sur tout son entourage français en Suisse, suspecté de menées anti-françaises et «bolcheviques" après 1917. Le capital de quelques quotidiens romands était en main française. Le matériel collectionné fut utilisé pour faire pression sur le gouvernement suisse en 1918, lorsqu'il s'agit de mâter une possible révolution en Suisse. Même si Rolland n'était pas directement visé, car trop connu, on écrémait tous ses contacts.

Cette surveillance se relâcha un peu avec l'armistice, l'abolition des pouvoirs spéciaux et, faute d'un service de police des étrangers au niveau fédéral, il n'y eut plus de harcèlement à ce niveau. Mais, dès 1923, progressivement, se mit en place un office fédéral de police coiffant les services cantonaux. Le canton de Vaud fut un des plus actifs. Les procédures d'octroi de séjour aux étrangers donnèrent lieu à la constitution de dossiers que le Ministère public fédéral dut avaliser - comme ce fut le cas pour Rolland. Avec la montée de la xénophobie et la peur du communisme, surtout après 1930, l'étau se resserra. Heureusement, ces fonctionnaires ne faisaient pas preuve de professionnalisme excessif: on en était encore loin de systèmes autoritaires comme ailleurs. Rolland était protégé par sa renommée et la sympathie de la gauche. Tant que Häberlin était en fonction à Berne, il n'avait rien à craindre au niveau fédéral. Malgré tout, on trouve dans des dossiers des Archives cantonales vaudoises des traces de plaintes et de délations que Berne enregistre, sans immédiatement prendre des sanctions, pour "étoffer»le dossier. On emmagasinait du "matériel compromettant» au cas où il fallait agir contre Rolland. La demande de visa de Maria Koudacheva, citoyenne soviétique, et le permis de séjour sollicité, mit Berne en alerte. On n'y voyait pas d'un bon œil le mariage avec Rolland qui fit d'elle une Française à qui l'on ne pouvait refuser le droit de domicile.

Dès 1935-1936 avec le nazisme aux frontières, la surveillance devint vitale, car les menées, voire même les assassinats politiques des polices italiennes, de la GPU soviétique et d'individus aux penchants héroïques 
(assassinat d'Otto Gustloff à Davos ou d'Ignaz Reiss à Lausanne) firent de la Suisse un terrain de combat ${ }^{11}$.

Le rigorisme administratif était, en revanche, très helvétique: pointilleux. Par exemple, en 1936, lorsque le Conseil fédéral, interdisant des publications "anti-religieuses et communistes", empêcha que Rolland ne pût se faire envoyer - même sous pli postal anonymisé l'Humanité. Cette mesure mesquine fut certainement une de ces gouttes qui firent déborder le vase et précipita la décision de Rolland de quitter la Suisse, en juin 1938.

Entrée en scène de Maria Koudacheva - un casse-tête bureaucratique aussi

On connaît les circonstances de l'entrée tardive vers 1928-1929 de la jeune veuve Maria Koudacheva dans la vie du bientôt sexagénaire Romain Rolland. On connaît aussi son rôle dans le rapprochement de Rolland avec l'URSS ${ }^{12}$. A Villeneuve ce fut un chamboulement de ménage, après la mort du père de Rolland: Maria s’installa dans la Villa Olga en évinçant Madeleine la sœur de Rolland. Celle-ci fut reléguée dans la Villa Lionette mitoyenne et en retrait de la Villa Olga. Dès lors, la préoccupation de la littérature orientale passa au second plan, au profit de celle de l'alliance soviétique pour combattre les fascismes - au prix d'un silence éloquent à l'égard de la montée stalinienne et des procès de Moscou.

Après une préparation du terrain par Georges Duhamel, le projet d'une édition complète des œuvres de Rolland en URSS servit de tremplin à Maria. Pour préparer cette édition, elle voulut se rendre chez Rolland - ce qui pour cette citoyenne soviétique (même d'ascendance française) fut un casse-tête, car la Suisse n'entretenait alors pas de relations diplomatiques avec l'URSS. Déjà l'obtention du visa pour un séjour temporaire à titre de "secrétaire» fut un tour de force. On trouve dans le fonds Rolland des Archives littéraires suisses à Berne un grand nombre de formulaires, rappels, demandes et télégrammes qui s'y réfèrent, traces de discussions âpres derrière les coulisses fédérales. Rolland mobilisa maintes fois le conseiller fédéral Häberlin qui resta affable,

II. Voir à ce sujet les études de P. Huber, dont Stalins Schatten in der Schweiz.

I2. Voir R. Rolland, Voyage à Moscou (juin-juillet 1935). 
mais fut certainement agacé par les demandes de son célèbre protégé. Par deux fois, Maria réussit en 1929 et 1931 à se faire accorder un séjour "de tolérance» limité qui tout en étant déclaré "professionnel» eut un caractère nettement touristique, si on ne veut pas parler de lune de miel. Ce dont Rolland ne se doutait pas, c'est la machinerie bureaucratique et policière qu'il déclencha. Le "tour de Suisse» de Rolland et Maria les conduisit par le Righi et les Alpes au bord du lac Majeur au Tessin. Ce qui signifie un passage par cinq cantons et un préavis de la police fédérale aux polices de chacun de ces cantons qui durent toutes accéder à la demande. De quoi occuper une vingtaine de fonctionnaires dans cinq cantons - car la police fédérale des étrangers ne dispose pas d'agents à elle sur le terrain, les cantons gardant cette chasse gardée jalousement pour eux ${ }^{13}$.

Ce casse-tête devint encore plus difficile, lorsque Rolland voulut obtenir un droit de "domicile permanent" pour Maria à Villeneuve dans les années 30. Il fallut beaucoup de pression et de "garanties» (garanties morales et des sommes considérables à déposer) pour que le canton de Vaud accordât un droit de domicile "de tolérance». On comprend pourquoi Maria se sentit constamment surveillée et pas forcément bienvenue dans le canton de Vaud - même si les Villeneuvois ne lui étaient pas hostiles.

Maria avait un fils adolescent de son premier mariage, Serge, qui fut éduqué en URSS. Rolland réussit à faire venir son fils adoptif en Suisse pour deux séjours, ce qui nécessita des démarches compliquées. Il respecta l'attachement de sa nouvelle épouse pour celui-ci. Comme l'a montré Bernard Duchatelet dans son livre sur le Voyage à Moscou de Rolland, cet attachement impliquait une retenue chez Rolland dans tout ce qu'il disait sur le régime stalinien - il en devint quasiment l'otage. On sait qu'il était très bien informé des dérives du régime dans la série des grands procès de Moscou, mais qu'il se tut. Il s'employa néanmoins à améliorer de façon discrète le sort de personnes en danger ${ }^{14}$. Ceci

13. En France aussi, avec ses nombreuses polices parallèles et spéciales, le processus de normalisation policière nationale était encore loin d'être accompli. Ce fut Vichy qui y réussit après 1940 .

I4. Il réussit néanmoins à faire libérer l'anarcho-syndicaliste et opposant Victor Serge (bien que celui-ci ne lui fut guère sympathique) et (temporairement) le célèbre médecin Oskar Hartoch (1881-1942) dont Rolland connaissait bien la sœur, Elsa Hartoch, qui fut une des fondatrices de l'Ecole internationale de Genève installée, 
explique ce sentiment d'ambiguïté de Rolland devant tout ce qui touche au stalinisme: à la lecture de son journal, on sait maintenant qu'il en dit moins qu'il pensait.

L'atmosphère politique en Suisse se gâta définitivement à partir de 1932: d'abord à cause de la crise économique mondiale et avec le revirement du Komintern en faveur d'un large "front antifasciste" (le mouvement du Congrès d'Amsterdam-Pleyel) et surtout en 1935 avec le Voyage à Moscou de Rolland. Les organisations clandestines du Komintern, pilotées par Willy Münzenberg et appuyées par Henri Barbusse, accaparèrent l'idée de Rolland en faveur d'un front de tous les partis contre les fascismes - un malaise qu'il ne confia qu'à son journal. Les organisateurs de ce congrès le mirent dans de mauvais draps : ils projetèrent de lancer le congrès le $1^{\text {er }}$ août 1932 à Genève, voulant profiter de la proximité de la SDN. Rolland eut toutes les peines à les en dissuader, car choisir cet endroit et cette date (fête nationale suisse) eût été une provocation du pays hôte. Ce choix fut éventé avant que Rolland ne put l'empêcher et provoqua un tollé contre Rolland en Suisse. Celui-ci eut toutes les peines à s'expliquer avec Häberlin. Le choix de Genève fut d'ailleurs contrecarré par une interdiction exprimée et par le gouvernement cantonal genevois et par le Conseil fédéral. Mais le mal était déjà fait. Rolland apparaissait aux yeux du public à la solde et aux commandes du Komintern.

Le sentiment d'être surveillé et seulement toléré s'amplifia chez Rolland qui, dès 1936, prit la décision de quitter la Suisse pour la France où s'était installé le Front populaire. A côté d'une France du Front populaire avec les espoirs qu'elle suscita, la Suisse de 1936 était un pays farouchement renfermé, anticommuniste et, aux yeux de Rolland, trop mou pour le danger fasciste. En outre, son protecteur Häberlin avait alors déjà démissionné. Pour Rolland, la neutralité suisse ne comportait plus les avantages qu'elle offrait entre 1914 et 1918.

C'est Maria qui emporta finalement la décision de quitter la Suisse: son statut d'ex-Russe avec droit de "domicile toléré », donc toujours

dans l'entre-deux-guerres, pour les fonctionnaires de la SDN. Après l'intervention de Rolland, on cessa temporairement de harceler Hartoch en URSS, mais à peine l'accord Ribbentrop-Molotov fut-il caduc, en juin 1941, à la suite de l'attaque de l'URSS par l'Allemagne, il ne fallut plus le ménager. Le NKWD se souvint des origines allemandes de Hartoch et le réemprisonna malgré son inébranlable loyauté soviétique. Hartoch fut exécuté en 1942. Voir à ce sujet l'étonnante notice biographique sur Oskar Hartoch par L. et S. Hasenson, «Die Familie Hartoch». 
provisoire, devait lui peser. Même la citoyenneté française acquise par le mariage avec Rolland à Villeneuve en 1934, n’apporta pas cette sécurité que lui offrait la France du Front populaire bien plus ouverte au communisme que la Suisse.

\section{Les anniversaires de Romain Rolland - indices de son impact}

Les années 1926 avec le $60^{\mathrm{e}}$ et 1936 avec le $70^{\mathrm{e}}$ anniversaire, tous deux fêtés à Villeneuve, révèlent un gradient dans la faveur du public en général, et en Suisse en particulier. Le Rolland de 1926 est encore célébré par un large public dépassant les clivages politiques. Celui de 1936 est également fêté, mais par un public exclusivement socialiste et communiste, par l'URSS et en Inde, au Japon et en Chine. Du côté germanophone il l'y eut plus que la Suisse alémanique et l'Autriche (grâce à Zweig) à rendre un hommage chaleureux, car le régime nazi l'avait proscrit dès 1934.

Le bilan de ces anniversaires montre que Rolland passait de plus en plus comme un partisan et non comme une figure d'intégration - ce rôle qui lui aurait tant plu. Il avait tant de fois lancé des appels, signé des manifestes, protesté et pris parti dans des cas politiques que l'on sent une certaine usure : ces manifestes n'eurent plus le même impact qu' "Au-dessus de la mêlée». On a l'impression d'un réflexe ou d'un automatisme dans ses prises de position qui deviennent schématiquement prévisibles, comme celles d'un Jean-Paul Sartre dans les années 60.

Il y eut un domaine où la démocratie suisse jouait en faveur de Rolland: le théâtre. Ses pièces étaient souvent interprétées en Suisse alémanique, mais, à partir de 1934, notamment à Zurich, ce fut le seul endroit où l'on pouvait voir ses pièces en allemand, notamment ses pièces sur la Révolution française. L'élite du théâtre allemand libre s'était exilée, repliée en Suisse, notamment au Schauspielhaus de Zurich devenu célèbre pour cela. Il n'y eut de scènes germanophones libres, à partir de 1933, que celles de Suisse et d'Autriche. Mais à partir de 1938, ce dernier refuge autrichien tomba aussi, de sorte qu'il ne resta que la Suisse pour jouer des pièces de Rolland en allemand. Montrer par exemple son Danton entre 1940 et 1945 était en Suisse un puissant symbole en faveur de la démocratie dans un pays vacillant au milieu d'une marée brune et noire. 


\section{Vézelay, refuge précaire - la guerre - le dilemme idéologique}

Quand les Rolland quittèrent Villeneuve, ils conservèrent la Villa Olga en location même après 1940. Romain subit l'accord RibbentropMolotov de 1939, qui aligna l'URSS avec le Reich, comme un choc qui atterra le monde communiste occidental: ce revirement de $180^{\circ}$ était difficile à avaler par ceux qui avaient lutté contre les fascismes et combattu en Espagne. Ce revirement le mit dans un dilemme: beaucoup de Français pouvaient douter de son patriotisme, car le PCF lutta contre l'entrée en guerre de la France. Grâce à sa renommée de philo-soviétique (même sans allégation directe au PCF, qui fut interdit en 1939), il devint auprès de l'occupant allemand ${ }^{15}$ un personnage à protéger en vertu de l'accord Ribbentrop-Molotov. Ce renversement entre le rôle des persécuteurs et des persécutés le protégeait temporairement de persécutions allemandes, mais n'était pas pour le rendre sympathique aux yeux du grand public. Le dilemme entre patriotisme avec acceptation d'une guerre qui s'annonçait et le pacifisme "défaitiste» des communistes fut un drame intime pour Rolland.

Bernard Duchatelet a étudié dans son Journal intime des années 1940 à 1944 ce volet de la vie intime de Rolland encore peu connu ${ }^{16}$. Il montre que Rolland, tout en ne cessant de placer son espoir en une URSS victorieuse, était déjà en désaccord avec le PCF dès 1938 et avait glissé d'un "pacifisme intégral» (comme celui de Giono) vers la nécessité d'une "acceptation raisonnée de la guerre», ce qui le conduisit à déclarer publiquement sa loyauté à la politique de Daladier. Rolland se trouva désemparé, profondément déçu et échaudé par le tournant des événements. Il cessa dès lors de s'exprimer en public (à plus forte raison que le fils de son épouse se battait comme soldat en URSS) et prit le chemin d'une émigration intérieure, se retirant de la scène politique et se consacrant à l'œuvre de Péguy et en renouant avec ses anciens camarades Paul Claudel et avec Alphonse de Châteaubriant.

I5. Vézelay se trouvait dans la zone dite "occupée», hors de la souveraineté directe de "l'Etat français» de Vichy, soumis au régime d'occupation de la Wehrmacht, néanmoins aux prises d'une administration civile aux ordres de Vichy.

I6. Le Journal des Années de Guerre II 1940-1944 (cité par la suite JAG II) est conservé au fonds Romain Rolland à la Bibliothèque de l'Université de Bâle et fut rendu public en 2004. B. Duchatelet a étudié l'année dramatique 1940: "Romain Rolland face à la Seconde Guerre mondiale». 
Dans le Journal de ces années, on retrouve un Rolland intime, bien plus secret que dans sa correspondance (qui était soumise à la censure) et étonnamment franc avec lui-même. Il réserve quelques surprises de taille à ceux qui s'attendent à un Rolland fidèle à son rôle d'intellectuel engagé de républicain de gauche et pacifiste. Selon Duchatelet, Rolland, confronté à la défaite, à la Révolution nationale de Pétain et au nouvel Etat français sans démocratie et aux garanties civiques largement abolies, s'est muté en "pétainiste» et "collaborationniste»! ${ }^{17}$ La déception a dû être grande pour un tel aveu, un tel changement d'attitude, même s'il ne dura pas au-delà de l'année 1942, période où le régime de Vichy perdit tout crédit et que les communistes résistants prirent le dessus. Ces derniers, Maria Romain-Rolland aidant, finirent par l'accaparer pour leurs besoins.

L'étiquette de "collaborationniste" étonne moins si on se réfère seulement à l'attitude de Rolland envers la Wehrmacht occupant le pays, du moins au début. Il semble que Rolland, qui profitait encore de son image de médiateur entre l'Allemagne et la France comme auteur du Jean-Christophe, ait redécouvert son ancienne ambition et ait voulu améliorer les relations avec l'occupant. Une "protection", toute relative, par l'occupant pouvait lui donner temporairement l'illusion de pouvoir jouer à nouveau ce rôle. Rolland alla même jusqu'à tancer les Allemands pour leur manque de psychologie qui les empêcherait d'obtenir une collaboration volontaire des Français. Hitler aurait eu le mérite de «nettoyer les écuries d'Augias»! 18

Entre juillet 1940 et juin 1941, la zone occupée était soumise au régime du Oberbefehlshaber Frankreich de la Wehrmacht (MBF), le général Otto von Stülpnagel et de son état major (auquel appartenait d'ailleurs l'ancien combattant et capitaine Ernst Jünger). Des mémoires

17. Nous adoptons la terminologie utilisée par l'historiographie récente: "pétainiste» désigne l'identification complète avec le programme politique de Pétain, tandis que «maréchalisme» signifie seulement la sympathie pour l'aura du maréchal dans un espoir - fallacieux - d'un chef d'Etat préservant le pays du pire. "Collaborationniste» est une attitude et option individuelle qui peut se placer dans ou hors de ce que l'on appelle la Collaboration d'Etat. Un François Mitterrand, par exemple, pouvait être qualifié de «maréchaliste», mais il passa à ce que l'on considère comme la "vichysso-résistance».

18. H. Speidel, Aus unserer Zeit, p. 186. 
du chef de cet état-major, le lieutenant-colonel Hans Speidel ${ }^{19}$, on sait que l'on prenait soin de "protéger» des artistes et des écrivains français, réputés "communistes» contre des représailles de Vichy qui n’avait jamais cessé de les combattre. La Wehrmacht découvrit Rolland dans son repaire de Vézelay qui devint bientôt un lieu de pèlerinage d'officiers lecteurs du Jean-Christophe. Des situations qui rappellent Le silence de la mer de Vercors - mais avec les rôles inversés. Il y eut d'autres personnages qui eurent des faveurs analogues, notamment Picasso, qui était resté à Paris et dont on ne pouvait ignorer son antifascisme et qui s'en tira indemne ${ }^{20}$. Pendant une courte durée, de 1940 à 1941, l'occupant essaya d'observer une attitude "militaro-légaliste", respectueux du droit des peuples en guerre, même sous un régime militaire, car Stülpnagel savait qu'en fusillant des otages - mesures imposées par Berlin - il provoquait plutôt une résistance qu'il ne la combattait ${ }^{21}$. Cette période prit fin dès juin 1941, lorsque le Reich attaqua l'URSS, que les communistes rejoignirent la Résistance et que des pouvoirs policiers allemands se substituèrent à la Wehrmacht. A partir de là, le SD (Sicherheitsdienst) allemand, en concordance avec une police française ambitieusement collaborationniste, prit les commandes en zone occupée et on n'eut plus besoin de se draper dans un légalisme militaire pour mâter le pays. Romain Rolland semble avoir bénéficié d'une certaine protection allemande (par exemple des services rendus ou des attributions de denrées) pour peu de temps.

19. Voir H. Speidel, Aus unserer Zeit (Speidel fut général de l'OTAN dans les années 50).

20. Traitement politique analogue à celui de Rolland par l'occupant allemand : voir M. C. Klepsch, Picasso und der Nationalsozialismus.

2I. D'où les précautions "légalistes" prises par un tribunal militaire réglementaire contre les résistants du Musée de l'Homme. Voir A. Hogenhuis-Seliverstoff, Des savants dans la résistance. Les nombreux actes d'exécutions d'otages étaient couverts par des «jugements», fussent-ils expéditifs - tant la Wehrmacht (avec sa Feldpolizei) tenait à son image légaliste. Les instances ultérieures, dépendantes du RSHA (Reichssicherheitshauptamt) (SD [Sicherheitsdienst], SS, Gestapo, entre autres) agissaient sur simples commandements, souvent oraux. 
La mémoire de Rolland dans la Suisse de l'après-guerre - revalorisation suivie du purgatoire de la guerre froide

En Suisse, la gauche n'avait jamais abandonné Rolland, notamment dans les milieux alémaniques où il apparaissait comme un phare de la démocratie et de la paix. Après 1943, après Stalingrad, même la Suisse officielle ne cessa de chercher le contact avec Rolland afin de lui faciliter un éventuel retour à Villeneuve. Pendant toute la guerre, Rolland regretta de ne pas être resté en Suisse: il rêva même de relancer un nouveau "Au-dessus de la mêlée», comme en 1914. Il restait l'oreille collée à la radio où il écoutait régulièrement entre autres les nouvelles de l'émetteur de la radio romande à Sottens ${ }^{22}$. Marie Romain-Rolland fut très active dans la conservation de sa mémoire et trouva en Suisse, entre 1948 et 1970, une "Association des Amis Suisses de Romain Rolland " 23 qui l'épaula. L'engouement pour ce Rolland retrouvé dura néanmoins à peine une décennie: avec la guerre froide beaucoup se désolidarisèrent. Cette fois, ce fut davantage la Suisse alémanique, plus encline à un certain "maccarthysme», qui renia le souvenir de celui qui s'était brûlé les ailes au stalinisme.

A long terme, l'engagement politique dans l'entre-deux-guerres nuisit à la réputation de Rolland en Suisse. L'éclat de son attitude pendant la Première Guerre mondiale se ternit au dam des grands espoirs que l'Europe pacifiste avait placés en lui après 1918. De figure d'intégration, Rolland passa au rôle de militant, voire de partisan, même si ce fut souvent involontairement et pour de bonnes raisons. "L'autre train», le stalinisme, que Rolland n'a pas vu venir ou pu critiquer publiquement a risqué de le broyer. Il semble qu'il incarna le type même du "compagnon de route» trahi par les siens, si bien décrit par François Furet.

Aujourd'hui encore, les interprétations de sa destinée politique sont polarisées: la vision d'un grand Européen et «compagnon de route», tragiquement accaparé par une illusion derrière laquelle se cache un système inhumain, concourt avec celle d'un homme qui serait allé jusqu'au

22. Où il pouvait suivre les chroniques politiques célèbres de René Payot. Ironie du sort, car Payot avait été un de ses principaux détracteurs dans la presse vaudoise.

23. Association active entre 1948 et les années 70, animée notamment par Charly Clerc et Sven Stelling-Michaud. Les actes en sont conservés aux Archives littéraires suisses de Berne. 
bout de ses convictions «révolutionnaires" même au moment le plus noir de l'histoire de son pays ${ }^{24}$. Ce clivage a aussi nui à sa fortune littéraire. En Allemagne pèse actuellement encore le souvenir d'un Rolland instrumentalisé par le régime de l'ancienne RDA - Rolland y reste dans le "purgatoire", malgré les efforts de la Gesellschaft der Freunde Romain Rollands in Deutschland. Dans l'Europe culturelle actuelle, pour la rénovation de laquelle il a tant lutté entre 1914 et 1918, on ne semble guère plus vouloir se souvenir de lui, alors que l'Union européenne économique s'est effectivement réalisée. En Inde, en Chine et au Japon, en revanche, sa mémoire est encore très vivante.

\author{
Jean-Pierre Meylan \\ Chercheur indépendant, Bâle
}

24. L'engagement politique de Rolland entre 1940 et 1944 a conduit à des interprétations différentes: J. Albertini (“Les dernières années 1939-1944», p. 107) conclut à un Rolland inébranlablement voué à un combat de la gauche jusqu'à sa mort tandis que B. Duchatelet, en se fondant sur le Journal, y voit un homme brisé, déçu, en émigration interne et se résignant au silence. 


\section{BIBLIOGRAPHIE}

\section{Source}

Rolland, Romain, Journal des Années de Guerre II 1940-1944, fonds Romain Rolland, Bibliothèque de l'Université de Bâle.

—, Voyage à Moscou (juin-juillet 1935): suivi de notes complémentaires (octobre-décembre 1938), éd. par Bernard Duchatelet, Paris, Albin Michel, 1992 (Cahiers Romain Rolland 29).

\section{Travaux}

Abraham, Pierre, Barrère, Jean-Bertrand, Buenzod, Janine, Chavanne, André, Reinhardt, Marc, Stelling Michaud, Sven, Romain Rolland, suivi de la correspondance inédite de Romain Rolland avec Adolphe Ferrière et Heinz Häberlin, Neuchâtel, A la Baconnière, 1969.

Albertini, Jean, "Les dernières années 1939-1944», Europe, 942 (2007), p. 105-116.

Duchatelet, Bernard, "Romain Rolland face à la Seconde Guerre mondiale: l'année 1940 (d'après son Journal inédit)", in Romain Rolland, une cuvre de paix, actes du colloque de Vézelay, 4-5 octobre 2008, éd. par Bernard Duchatelet, Paris, Publications de la Sorbonne, 2010, p. 181-194.

Hasenson, Leonid et Sofia, «Die Familie Hartoch - eine Geschichte in Russland und in der Schweiz", Schweizerische Zeitschrift für Geschichte, 51 (2001), p. 106-110.

Hogenhuis-Seliverstoff, Anne, Des savants dans la résistance. Boris Vildé et le réseau du Musée de l'Homme, Paris, CNRS, 2009.

Huber, Peter, Stalins Schatten in der Schweiz, Zürich, Chronos, 1994. 
Klepsch, Michael Carlo, Picasso und der Nationalsozialismus, Köln, Patmos Verlag, 2007.

Mattioli, Aram, Gonzague de Reynold: un idéologue suisse autoritaire, Fribourg, Editions universitaires, 1997.

Meylan, Jean-Pierre, La "Weltbibliothek" et la Maison des Amis, un projet de Romain Rolland (Villeneuve VD, Calcutta et Bâle, 19221926), Brèves, Association Romain Rolland, 2009 (Etudes Rollandiennes 21).

—, "Romain Rolland au-dessus, mais aussi dans la mêlée», in Romain Rolland, une cuvre de paix. Actes du colloque de Vézelay, 4-5 octobre 2008, éd. par Bernard Duchatelet, Paris, Publications de la Sorbonne, 2010, p. 73-87.

—, «Der Plan einer Weltbibliothek von Romain Rolland und seinem Schweizer Verleger und Mäzen Emil Roniger, 1922-1926", in LIBRARIUM, fasc. 1 (avril 2010), p. 3-13.

Schütт, Julian, Germanistik und Politik, Zürich, Chronos, 1996.

Speidel, Hans, Aus unserer Zeit: Erinerungen, Berlin West, Propyläen Verlag, 1977.

Vallotton, François, Ainsi parlait Carl Spitteler, Lausanne, Université de Lausanne, 1991 (Histoire et société contemporaines 11). 
